\title{
LA AUTOESTIMA DE LAS MUJERES EN UN MUNDO PATRIARCAL Y MECANISMOS DE FORTALECIMIENTO: ESTADO DE LA CUESTIÓN ${ }^{1}$
}

\author{
Carmen del Socorro Medina Sarmiento ${ }^{2}$
}

\begin{abstract}
La autoestima de las mujeres en un mundo patriarcal y mecanismos de fortalecimiento: estado de la cuestión

Resumen: la autoestima es un concepto fundamental de la subjetividad, pero su abordaje se ha hecho principalmente desde una perspectiva individualista, obviando la pertinencia del análisis de género. El presente trabajo presenta por qué este análisis es fundamental y recoge el estado de la cuestión para obtener claves que permitan el fortalecimiento de la autoestima de las mujeres en aras del empoderamiento feminista desde una perspectiva interseccional.

Palabras clave: autoestima, feminismo, interseccionalidad, subjetividad, empoderamiento.

Women's Self-Esteem in a Patriarcal World and Strengthening Mechanisms: State of the Question.

Abstract: self-esteem is a fundamental concept of subjectivity, but its approach has been mainly from an individualistic perspective, ignoring the relevance of gender analysis. The present work, adopting an intersectional perspective, presents why this analysis is fundamental and collects the state of the question to obtain clues that allow for the strengthening of women's self-esteem and for the feminist empowerment.

Key words: self-esteem, feminism, interseccionality, subjectivity, empowerment.
\end{abstract}

\section{Introducción}

El principal objetivo del presente trabajo es desentrañar estrategias para fortalecer la autoestima de las mujeres desde una óptica feminista. La necesidad de este abordaje surge de la insuficiencia de análisis en psicología de cómo el sistema patriarcal puede interferir de forma específica en la autoestima de las mujeres. Hasta el momento, la psicología ha hecho una definición generalista y ambigua de la autoestima (Ortega, Mínguez y Rodes 2001: 52) y además no ha tenido en cuenta la estructura social en su sentido sociológico. Esto hace necesario conceptualizar la autoestima a luz de su

\footnotetext{
${ }^{1}$ Fecha de recepción: 13/04/2018.

Fecha de aceptación: 06/08/2018

${ }^{2}$ Licenciada en Psicología por la Universidad de La Laguna, Máster en Género e Igualdad en Ámbito Público y Privado por la Universidad Jaume I. Técnica de sensibilización en la ONG Médicos del Mundo Canarias; $\square$ carmenmedsar@gmail.com.
} 
influencia por el sistema patriarcal, de cara a poder realizar propuestas de intervención aterrizadas a la realidad específica de las mujeres.

Para ello, en primer lugar, se pone en evidencia la necesidad de entender la influencia que la desigualdad de género tiene sobre la autoestima de las mujeres y también de tener en cuenta la perspectiva interseccional. Posteriormente se aborda el estado de la cuestión en tres sentidos: 1. Cómo define la psicología la autoestima y qué elementos ha identificado que la influyen; 2. Qué variables ha identificado el feminismo que afecten a la autoestima de las mujeres y 3. Qué mecanismos de fortalecimiento de la autoestima se conocen desde la bibliografía feminista.

En las conclusiones se realiza una recogida de aspectos identificados en el estado de la cuestión para construir una propuesta conceptual de la autoestima que permita abordarla desde una óptica feminista, haciendo del trabajo específico de la autoestima un mecanismo de reparación de las heridas patriarcales en las mujeres.

\section{Marco teórico}

2.1. Perspectiva de género: la necesidad de tenerla en cuenta

El presente trabajo se enmarca en la teoría política feminista, es decir, reconoce que habitamos una estructura social patriarcal y nos situamos críticamente en el rechazo de ésta, generando propuestas y praxis de cambio.

El término patriarcado fue definido por Kate Millet en 1969 "como una institución en virtud de la cual una mitad de la población (es decir, las mujeres) se encuentra bajo el control de la otra mitad (los hombres)" (Millet 1995: 70).

Se trata de un sistema social que establece diferenciaciones en cómo han de ser y a qué se han de dedicar mujeres y hombres y jerarquiza dicha diferenciación, de modo que el valor social que tienen las características y roles asociados a los hombres (características y roles masculinos) son mejor valorados que los asociados a las mujeres 
(características y roles femeninos), adquiriendo mayor visibilidad y siendo tomados como medida de todas las cosas (androcentrismo). Un ejemplo destacado de como este falso universal de lo masculino influye en nuestra concepción del mundo es la teoría del desarrollo moral que propuso Lawrence Kolhberg, afirmando que las mujeres se estancan en un estadio inferior por ser moralmente más débiles. Carol Gilligan, discípula de Kolhberg, revisó los estudios de su maestro y evidenció que sus conclusiones estaban sesgadas porque él había hecho únicamente la teoría del desarrollo moral de los hombres y pretendía generalizarla a las mujeres sin tener en cuenta que la estructura patriarcal influye la subjetividad de hombres y mujeres distintamente (Fasciolli 2010: 42). El impacto que este sistema tiene en la autoestima de las mujeres lo señala Marcela Lagarde afirmando que vivir en condiciones patriarcales daña la autoestima de todas las mujeres, y que ello requiere atención específica (Lagarde 2000).

La histórica invisibilización de las mujeres en todas las esferas, la mayor pobreza de las mujeres respecto a los hombres, el índice de violencias machistas y la escasez de mujeres en puestos de decisión (aun en aquellas esferas feminizadas) son solo algunos de los múltiples indicadores del impacto del sistema patriarcal en la vida de las mujeres y de la necesidad de la perspectiva de género en cualquier campo de estudio, y más aún en los que abordan directamente las cuestiones humanas. Crecer en este sistema social desigual cala en nuestra subjetividad mediante el proceso de socialización (Bardwick 1986: 255; Steinem 1995: 337). Los seres humanos crecemos y nos configuramos en relación con las otras personas, de modo que los valores sociales influyen en todos los aspectos de nuestro Yo. De este modo, la socialización de género, en tanto en cuanto sitúa a las mujeres en posición de dominación, supone una forma de violencia simbólica (Bourdieu 2016: 49). Atender específicamente los elementos de la subjetividad es una estrategia para revertir los efectos de esta forma de violencia sobre las mujeres, y por ello es fundamental el análisis feminista de la autoestima.

\subsection{Interseccionalidad}

No obstante, este mecanismo de universalizar la experiencia de los grupos dominantes ha sido reproducido en el seno del propio feminismo, dando por 
generalizable la experiencia de las mujeres blancas de clase media. Esto fue puesto en evidencia especialmente por parte de feministas negras como Ángela Davis (2004), quien analizó la confluencia de género, raza y clase, concluyendo que es necesaria una mirada integral de las formas de opresión para construir estrategias políticas realmente inclusivas.

Este apunte permite introducir un concepto de reciente creación en los estudios feministas y de género: la interseccionalidad. Dicho concepto fue acuñado por Kimberlé Crenshaw en la década de 1980, para referirse a un enfoque en las ciencias sociales que contemple otras fuentes de opresión aparte de la construcción del género, tales como la etnia, la orientación sexual, la clase, etc. (Salem 2014: 115). Ahora bien, aunque no hubo un término que designase esa perspectiva hasta que no nos fue dado por Kimberlé Crenshaw, sí que la necesidad de tener en cuenta múltiples ejes de opresión además del género ya existía, principalmente en el feminismo negro, como se ha señalado anteriormente.

Para evitar estos falsos universales y la exclusión de las personas puestas estructuralmente en situación de opresión es por lo que se hace indispensable el abordaje de la autoestima desde una perspectiva feminista interseccional. El análisis feminista interseccional es una herramienta política y metodológica para la justicia social que nos permite trazar estrategias para la igualdad que sean realmente efectiva para todas las mujeres.

\subsection{Subjetividad y transformación social: una relación bidireccional}

No obstante, la relación entre la estructura social y la subjetividad no es unidireccional. Si bien las variables sistémicas influyen enormemente en la configuración del Yo, es posible transformar los elementos de la subjetividad hacia formas más coherentes con el sistema social que queremos construir. Esto supone romper con la perspectiva individualista, como se ha dicho, pero también con una óptica sociologizante que reduzca a las personas a agentes pasivos en el proceso de socialización. 
La relación bidireccional entre sistema social y subjetividad aterrizada al análisis de la autoestima se muestra de forma clara en su relación con un elemento central en el feminismo, y en general en todos los movimientos sociales que parten del reconocimiento de una opresión sistémica hacia un grupo determinado: el empoderamiento. De este concepto existen varias definiciones, coincidiendo todas ellas en señalar tanto una dimensión individual de toma de conciencia de la opresión sufrida como una dimensión colectiva de movilización y transformación de las estructuras de poder (Cano y Arroyave 2014: 99). Sobre la autoestima no hay tampoco una definición única (Ortega, Mínguez y Rodes 2001: 47) y aunque hay gran diversidad teórica sobre sus elementos componenciales a rasgos muy generales podría definirse como el afecto hacia una/o misma/o.

Al situarnos el patriarcado a las mujeres en posición de inferioridad e interiorizar esa inferiorización, la autoestima se convierte en un elemento fundamental para el empoderamiento, pero no es el empoderamiento. El empoderamiento necesita la autoestima, pero también la canalización de esa subversión de la subjetividad patriarcal de las mujeres hacia la transformación colectiva de la estructura social. Así, la autoestima, aunque se genera en interacción con un sistema determinado, es un elemento individual.

Es posible que una mujer mejore su autoestima y no lo hagan otras mujeres. Sin embargo, las consecuencias del empoderamiento siempre son colectivas. Es posible, por ejemplo, que una mujer pierda el miedo aprendido a hablar en público, segura de que puede hacerlo bien y de que tiene derecho a ocupar ese espacio, pero sin embargo no sea escuchada en la misma medida que sus homólogos masculinos. El empoderamiento, sin embargo, tendría lugar con la transformación a nivel social de los estereotipos que impiden tomar tan en serio como a los hombres a las mujeres en los espacios de palabra. La autoestima supone revertir los efectos de la violencia simbólica en las mujeres, mientras que el empoderamiento supone la abolición, o al menos la paliación de las violencias sobre las mujeres.

No hay consenso en lo investigado hasta ahora acerca de si amoldarnos las mujeres a las expectativas patriarcales mejora la autoestima. Marcela Lagarde afirma que sí (2000: 33), mientras que para Emilce Dio Bleichmar amoldarse a los estereotipos 
de género, paradójicamente, no supone una fuente de autoestima, sino que apuntala a una mayor probabilidad para la depresión (1993: 273). Desde la óptica feminista lo que interesa es el empoderamiento, es decir, la transformación subjetiva y social de lo injusto del sistema en pro de la igualdad y libertad de las personas oprimidas. En este sentido el interés por la autoestima es tal en tanto en cuanto la autoestima es un elemento fundamental del empoderamiento y desafía la subjetividad patriarcal de las mujeres en aras de subjetividades más congruentes con los cambios estructurales que queremos ver materializados.

Que cada vez más mujeres fortalezcan su autoestima al margen de la subjetividad patriarcal supone visibilizar que diferentes formas de ser mujer son posibles. Serían referentas para otras mujeres y para la sociedad en su conjunto, y es también un paso fundamental para la movilización conjunta que favorezca los procesos de empoderamiento. Sin la toma de conciencia sobre la opresión sufrida y la legitimación del propio Yo no se hace posible esa movilización conjunta.

\section{Estado de la cuestión}

3.1. La autoestima desde la psicología: la ausencia de la perspectiva de género

A pesar de que la autoestima es un concepto ampliamente empleado y estudiado en la psicología, no existe una sistematización de sus elementos clave que nos permita diseñar intervenciones específicas. Esto se debe a que ha sido considerada un elemento instrumental para determinadas variables psicológicas como el rendimiento, sin que se le haya dado valor en sí misma, tal y como señalan Pedro Ortega, Ramón Mínguez y María Luisa Rodes (2001: 46). En su revisión sobre las definiciones de la autoestima encuentran ambigüedad y un uso indiferenciado de este concepto como sinónimo de otros, tales como autoajuste o autoconcepto, aunque hay consenso en considerarla el aspecto valorativo del propio Yo y en reconocer la influencia que las otras/os tienen en 
ella. Sin embargo, es importante señalar que esta dimensión relacional es vista meramente en términos interactivos, sin tener en cuenta como esas interacciones humanas están influidas por la estructura social.

El autor más conocido en el estudio de la autoestima es Morris Rosenberg, quien, aunque fue sociólogo, diseñó un instrumento de medida de la autoestima que es la herramienta clínica más empleada para ello por la fiabilidad y validez que ha mostrado y la sencillez de su aplicación, dado que consta únicamente de diez ítems. No obstante, no tiene en cuenta la autoestima desde una perspectiva de género y si bien ese instrumento pueda identificar una baja autoestima, no nos habla de las causas en términos estructurales.

Un esfuerzo por discernir elementos constituyentes de la autoestima es el llevado a cabo por Patricia Linville en su conocido artículo "No pongas todos los huevos en la misma cesta", donde crea el concepto de complejidad del Yo para referirse a la cantidad de ámbitos de desarrollo personal. Restringir el número de esferas vitales hace que si nuestro desempeño en alguna de ellas va mal en algún momento, ello influya en nuestra autoestima mucho más que si tenemos otros ámbitos de los cuales obtener un feedback positivo de nosotras/os mismas/os (Linville, 1985).

Otro aspecto constitutivo de la autoestima lo señala Judith Bardwick (1986), al señalar que la autoestima será mayor cuanto mayor sea la similitud entre el Oo real $^{3}$ y el Yo ideal, o bien de cuanto alcanzable sea el Yo ideal. Esta autora hace un análisis de como estos elementos se ven afectados específicamente en las mujeres, por lo que se desarrollará más extensamente al recoger el estado de la cuestión desde los estudios feministas.

Así, la conceptualización de la autoestima se ha hecho en términos generales como "estima del Yo", sin que se hayan sistematizado sus elementos constituyentes. En los escasos trabajos donde se ha tratado de desentrañar qué variables concretas la influyen, ha sido reduciéndola a una única dimensión. Además, es particularmente escasa la bibliografía que aborda la cuestión desde la perspectiva de género. Todo esto no nos permite comprender profundamente como se configura y como podrían

\footnotetext{
${ }^{3}$ El Yo ideal es lo que nos gustaría llegar a ser, mientras que el Yo real es lo que somos.
} 
generarse herramientas psicoterapéuticas para su fortalecimiento, lo que hace necesario contar con un análisis de cómo se configura patriarcalmente la autoestima de las mujeres y con una definición de autoestima que nos permita apresar sus elementos clave para el trabajo de esta, desde una perspectiva feminista interseccional.

3.2. La autoestima desde el feminismo: los elementos que la influyen

Quien más ha desarrollado el análisis de la autoestima de las mujeres desde una óptica feminista ha sido Marcela Lagarde, quien ha escrito un libro completo sobre ello titulado Claves feministas para la autoestima de las mujeres (2000). Define la autoestima como:

Una dimensión de la autoidentidad marcada por todas las condiciones sociales que configuran a cada mujer y, de manera fundamental, por la condición de género. Conformadas como seres-para-otros, las mujeres depositamos la autoestima en los otros y, en menor medida, en nuestras capacidades. La cultura y las cotas sociales del mundo patriarcal hacen mella en nosotras al colocarnos en posición de seres inferiorizadas y secundarias, bajo el dominio de hombres e instituciones, y al definirnos como incompletas. (Lagarde 2000: 32)

Muestra de qué forma se configura la autoestima en el seno del patriarcado, poniendo énfasis en las contradicciones internas que genera el que las mujeres actualmente se encuentren "entre lo público y lo privado, entre la tradición y la modernidad" (Lagarde 2000: 36), donde se resalta la independencia económica, el trabajo fuera de casa, la autonomía, y al mismo tiempo el seguir siendo para otras/os, los cuidados, la belleza. A ese cúmulo de exigencias las llama sobremodernidad. Estas contradicciones se materializan en la subjetividad de las mujeres con lo que la autora llama sincretismo genérico y escisión. El sincretismo genérico es una autoestima marcada tanto por la infravaloración, la inseguridad, el temor, y la dependencia respecto a otras/os, como por la exaltación y valoración del ajustarse a mandatos patriarcales, y al mismo tiempo la valoración de la independencia y las cualidades personales. Todas 
estas contradicciones internas llevan a sentir escisión en al menos algunos momentos de la vida, es decir, a la disyuntiva entre el ser para sí y ser para otras/os (2000: 36-38).

También Gloria Steinem ha abordado la autoestima de las mujeres desde una perspectiva feminista en La revolución desde dentro (1995). Identifica algunos elementos de la socialización patriarcal que considera claves, como son los cánones de belleza. Sobre los mismos señala que si bien existen modelos de belleza masculinos, los hombres son valorados por el éxito profesional y no por su capacidad de atracción, como sí es el caso de las mujeres. Los cánones de belleza no se caracterizan solo porque sea necesario que los alcancen las mujeres, sino también por su inaccesibilidad, lo que produce una insatisfacción generalizada de las mujeres hacia sus cuerpos, independientemente de que éstas se ajusten más o menos a los estándares de belleza, que deriva en problemas de salud tan serios como los trastornos de la conducta alimentaria. El desconocimiento de que los cánones de belleza son un constructo cultural y político y la impresión de que son un absoluto universal produce culpabilidad y la sensación de que en las mujeres que no los alcanzan hay algo anómalo. Señala también la influencia de la raza en estos cánones de belleza, siendo además más inferiorizadas las mujeres que no son blancas, pues solo éstas pueden llegar a ser consideradas hermosas. (1995: 286-290). Sobre los trastornos de la conducta alimentaria expone además que no son algo propio solo de las mujeres a quienes se diagnostican, sino que en ellas se da de una forma más marcada algo que le pasa a un número de mujeres mucho mayor (1995: 299). Otra clave identificada por la autora es la restricción de características manifestables por las mujeres. Se atribuyen a unas y a otros características diferentes, y a las mujeres nos toca un espectro mucho más limitado de formas de ser. Además, aquellas características aceptables en las mujeres están inferiorizadas respecto a las masculinas, siendo menos deseables (1995: 337).

Sobre la influencia del aspecto físico en la autoestima también dan cuenta Marika Tiggeman y Claire Stevens (1999: 103), quienes realizaron un estudio intergeneracional y encontraron que a mayor peso menor autoestima en las mujeres de 30 a 49 años, pero no en las más jóvenes o mayores. Coincidiendo con esa construcción de Lagarde sobre las mujeres como seres para otros, que puede verse también en el hincapié que hace Steinem en el atractivo de las mujeres, es central en mucha bibliografía feminista la idea 
de que las mujeres nos vemos a través de la mirada masculina, si bien es bibliografía que no aborda la autoestima directamente. Así, en La mujer rota (1988), Simone de Beauvoir expone tres relatos de mujeres que han construido su autoestima en base a la imagen que sus familias tenían de ellas, especialmente sus parejas masculinas. Así, tras la ruptura con estas personas sienten la pérdida de identidad, no saben cómo definirse. Es éste el mismo problema que plantea Betty Friedan en la Mística de la feminidad, que da cuenta del malestar que sienten las mujeres al verse abocadas a ser madres y esposas, renunciando a la consecución de proyectos propios. La autora halla la clave del malestar de las mujeres abocadas al hogar en la psicología que plantea una tendencia natural al crecimiento y desarrollo natural del ser humano. Esta posibilidad es anulada en las mujeres al quedar confinadas al mundo doméstico, cuestión que la psicología no ha señalado porque cuando habla de tendencia hacia la autorrealización piensa en los hombres y considera normal que las mujeres no la desarrollen:

\begin{abstract}
Si estas nuevas teorías son acertadas y si el impulso del ser humano fundamental es, no la necesidad imperiosa del placer o de la satisfacción de las necesidades biológicas, sino la necesidad de crecer y desarrollar el potencial pleno de cada persona, sus días cómodos y vacíos y sin propósito son un buen motivo para sentir un terror sin nombre. En nombre de la feminidad, han evadido las opciones que les habrían dado un propósito personal, un sentido de su propio ser (Friedan 2017: $377)$.
\end{abstract}

Mediante una serie televisiva de gran éxito, "Modos de ver", que luego se convertiría en ensayo, John Berger establece una comparación entre los roles y estereotipos sociales de las mujeres, y su representación en el arte pictórico, con especial énfasis en el desnudo. Desde su análisis, las mujeres aparecen en las pinturas tal y como se espera que sean en la sociedad: sumisas, entregadas, definiéndose a sí mismas mediante la mirada de los hombres que la observan, pues siempre aparece consciente de que está siendo observada (Berger 2016: 25).

También Liliana Mizrahi afirma que se nos atribuyen características y obligaciones en base a las necesidades de los otros, utilizando socialmente la culpa como un mecanismo de control de las mujeres, que nos lleva a desconfiar de nosotras mismas, de nuestros conocimientos y aceptar servilmente que "[...] nuestro espacio, 
nuestro rol y nuestras obligaciones han sido atribuidos en función de "las-necesidadesde-los-otros". Los otros: esferas divinas ante las cuales, en realidad, somos seres abandonados" (Mizrahi 1990: 28). De este modo Liliana Mizrahi dice que no solo es que las mujeres estén marcadas por el hecho de que su rol vital sean los cuidados, sino también por la falta de reconocimiento.

Judith Bardwick (1986: 259-260) afirma también que la principal motivación de las mujeres es la motivación de filiación. Se enseña a las mujeres a estimarse por logros académicos, pero también y principalmente por la afiliación con un hombre, lo que pasa a ser la motivación principal en su adolescencia y adultez temprana, y su principal fuente de autoestima. Así, coincide con Steinem al decir que a las mujeres se les reduce mucho los roles a desempeñar y las características a desarrollar. Esta restricción de sus características manifestables produce que sientan que su Yo ideal no puede realizarse.

En la misma línea, María Asunción González de Chávez Fernández (1993: 103) afirma que a las mujeres socialmente se las presiona para que reduzcan todos sus roles a los cuidados, de forma tal que en su subjetividad solo esos roles forman parte de su identidad deseada, inhibiendo cualquier deseo que no se corresponda con ello. Esto unido a que su necesidad prioritaria es el amor y que sienten que si tienen éxito profesional o reconocimiento en otras áreas su relación se vería perjudicada, hace frecuente renunciar a los deseos que se alejan del rol patriarcal, y sentirse constantemente abatidas por la culpa si no lo hacen.

Sin embargo, se ha cuestionado hasta qué punto esta configuración de la subjetividad de las mujeres es una experiencia universalizable. Según María del Pilar Cruz Pérez (2012: 63), precisamente la causa de la baja autoestima de las mujeres con diversidad funcional ${ }^{4}$ es el ser consideradas incapaces de cubrir los roles esperados para las mujeres (madre, ama de casa y dispensadoras de cuidados), lo que hace que se sientan inferiores tanto a los hombres con diversidad funcional como a las mujeres sin diversidad funcional. Critica además que dentro del propio feminismo se ha puesto poca atención a estas mujeres, y que cuando se ha hecho ha sido mostrándolas como víctimas de esa doble opresión, pero no visibilizando sus propias voces y experiencias como

\footnotetext{
${ }^{4}$ Se emplea este término por ser el que actualmente se utiliza de forma más extendida entre los trabajos anticapacitistas.
} 
sujetas activas y haciendo visibles ejemplos de subversión. La misma preocupación muestra Marta Senent Ramos (2014: 181), quien además cuenta que las mujeres con diversidad funcional sufren hasta cinco veces más abusos sexuales que el resto de mujeres, con la paradoja de que luego son consideradas asexuales e incapaces de atraer sexualmente a alguien, lo que contribuye a invisibilizar aún más el problema. Marta Senent pone el énfasis en la visibilización de referentas como herramienta de transformación, mostrando ejemplos de mujeres con diversidad funcional que han subvertido esa construcción mostrándose sujetas de deseo y poder de seducción.

También en los feminismos negros se ha criticado esa pretensión de universalidad de una visión de la subjetividad de las mujeres que realmente no representa a las mujeres negras. Según Mercedes Jabardo (2012: 29) en los feminismos negros existe la necesidad de resignificar el término "mujer", ya que las mujeres negras eran construidas como "no mujeres", estando esta identidad construida desde el feminismo blanco. Ejemplifica para ello el discurso “¿Acaso no soy yo una mujer?”, que Sojourner Truth pronunció en la Convención de los derechos de la mujer en 1851, en el que exaltaba su capacidad para hacer tanto lo asociado a los hombres (el trabajo de labranza), como aquello asociado a las mujeres blancas (la maternidad), y reivindicaba que sin embargo no tenía los mismos derechos ni que unos ni que otras.

bell hooks ${ }^{5}$, a la citada obra de Friedan La mística de la feminidad, le reprocha que haya servido para representar únicamente a las mujeres blancas de clase media. Al decir Betty Friedan que hay una voz en las mujeres que dice querer algo más que marido, hijos y la vida del hogar para referirse a ese "algo más" como a una carrera, no hace mención a quienes serían entonces las personas que cuidasen de sus hogares. bell hooks critica esa presunción de universalización de los problemas de las mujeres blancas de clase media, y en sus propias palabras:

Muchas mujeres vivían preocupadas por la supervivencia económica, la discriminación racial y étnica, etcétera. Cuando Friedan escribió "La mística de la feminidad", más de un tercio de las mujeres formaban parte de la fuerza de trabajo. Aunque muchas mujeres deseaban ser amas de casa, sólo quienes tenían tiempo

\footnotetext{
${ }^{5}$ Se emplea la minúscula respetando el deseo de la autora, quien escribe su nombre en minúscula para destacar que lo importante de sus textos es su contenido, no quien es ella.
} 
libre y dinero podían formar sus identidades a partir del modelo de la mística femenina. (hooks 2004: 34)

Julie Ann Mckullin y John Cairney (2003:75) han explorado la intersección entre edad, clase, género y autoestima, encontrando que en todos los grupos las mujeres presentan una autoestima menor que los hombres. No encontraron diferencias entre autoestima según clase social en la juventud, pero sí en edades posteriores, y también hallaron que, a mayor edad, menor autoestima.

3.3 La autoestima desde el feminismo: Experiencias de fortalecimiento

Es importante no ver solo la bibliografía que analiza cómo se construye en el patriarcado una autoestima pobre en las mujeres, sino también conocer cuáles son las experiencias de fortalecimiento que se han puesto en marcha. Esto es especialmente relevante para legitimar la capacidad de agencia de las mujeres y para contar con herramientas para trabajar la propia autoestima, trasladando a más mujeres esas experiencias de fortalecimiento. Así, aunque poca y brevemente desarrollada podemos encontrar bibliografía que señala como la conciencia feminista mejora la autoestima de las mujeres.

Ana de Miguel (2016: 31) afirma que la militancia feminista hace que las mujeres se enfrenten explícitamente a su condición de segundo sexo ${ }^{6}$ y a los miedos construidos en el patriarcado, y las lleva a reconocerse como personas independientemente de estar o no emparejadas con un hombre. Con el afrontamiento de los miedos patriarcales dentro del activismo feminista coincidía ya también Marcela Lagarde, quien cuenta en su ya nombrado trabajo que en la tradición feminista ha sido fundamental para las mujeres compartir experiencias en pequeños grupos no mixtos, ya que ello permite, además de afrontar miedos, oír sus propias voces, dudar, aprender y valorar sus cualidades y logros, todo ello desde la conciencia de género, impulsando estos grupos cambios sociales extensivos en muchas ocasiones, pero especialmente en sus entornos

\footnotetext{
${ }^{6}$ En alusión a "El segundo sexo", libro escrito por Simone de Beauvoir en 1949.
} 
cercanos y en sus propias vidas (2000: 66-67). Para esta autora el feminismo es fuente de autoestima ya que conocer la genealogía feminista hace que las mujeres se sientan parte de ello, encuentren una ubicación subjetiva en el mundo en relación con otras de las que se aprende y se heredan poderes y derechos.

Cuenta como cada vez más mujeres se definen por la fortaleza, después de sentir la escisión superan sus contradicciones subjetivas y se definen por sí mismas y desde ahí se relacionan con las/os otras/os, se valoran a sí mismas y no se posicionan en subordinación. De ellas comenta Lagarde que no se trata solo de mujeres acomodadas, sino de mujeres de todas las condiciones socioeconómicas, de diferentes culturas y etnias y las sitúa como referentas:

\begin{abstract}
Son mujeres libres aunque la sociedad no legitime sus libertades. De mujeres así se nutre la teoría feminista de la autoestima. De ellas aprendemos maneras de enfrentar experiencias adversas o complejas, en las que despliegan la creatividad e inventan alternativas prácticas que devienen en solidez personal (Lagarde 2000: 39).
\end{abstract}

También habla de aquellas mujeres que teniendo en cuenta el daño a la subjetividad que hace vivir en condiciones patriarcales no se ajustan a los mandatos de género, a quienes llama asintónicas, y quienes pueden tanto sentirse abrumadas por ello como hacer de su asintonía un proceso de crecimiento personal, autoafirmación y fortalecimiento. Este proceso se genera especialmente con el conocimiento de la existencia de más mujeres que así se sienten y que han escogido una forma de vivir su vida coherente con su sentir, es decir, a partir de las referentas.

María Asunción González de Chávez, Carmen González Nogueras y Lucía Valdueza (1993: 281) cuentan su experiencia en el trabajo con un grupo de mujeres con sintomatología de cuadro depresivo, aportando claves sobre cómo opera en las mujeres el trabajo de la subjetividad desde una óptica feminista. Resaltan, como Marcela Lagarde, que la propia identificación de género en un grupo de mujeres donde sentirse escuchadas es fuente de autoestima. Asimismo, resaltan que el poder explicar sus malestares desde el feminismo, conociendo que las inhibiciones de sus deseos tienen 
que ver con la socialización de género, les permite autoafirmarse, protestar, ser permisivas consigo mismas y comenzar a buscar gratificaciones personales.

Según encontraron Marika Tiggeman y Claire Stevens en su citado estudio entre la relación del aspecto físico en la autoestima de las mujeres, esta influencia del peso en la autoestima de las mujeres de 30 a 49 años deja de serlo cuando existen actitudes feministas (Tiggeman y Stevens 1999: 103).

Aurora Levins Morales cuenta que su "descubrimiento de una comunidad de escritoras, artistas y pensadoras feministas de color, fue probablemente la más profunda legitimación que nunca haya recibido de mi derecho a existir, a conocer, a nombrar mi propia realidad" (2004: 66).

Gloria Steinem afirma que el conocimiento de cómo se genera culturalmente el concepto de belleza y como se ha articulado para oprimir a las mujeres minimiza el efecto de los cánones y fortalece la autoestima (Steinem 1995: 284).

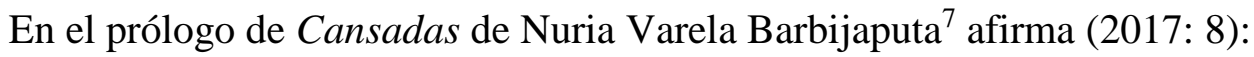

Lo nuestro (el feminismo) está siendo tan poco a poco que no advertimos que el feminismo nos había estado armando hasta los dientes con escudos y lanzas ingrávidos de los que ya no podríamos desprendernos ni queriendo, y eso nos hace caminar más seguras, alzar la barbilla, mirar a los ojos, poco a poco. (Varela, 2017: 8)

Así, la propia toma de conciencia, el conocimiento de la fuente de los malestares se convierte en sí misma en una herramienta para el fortalecimiento de la autoestima.

\section{Conclusiones}

De lo visto hasta el momento y teniendo en cuenta la ausencia de bibliografía que aborde la cuestión específicamente desde una óptica interseccional, se pueden extraer

\footnotetext{
7 Pseudónimo de una ciberactivista feminista que ha preferido mantener su anonimato dadas las constantes amenazas que recibe en redes sociales a razón de su activismo.
} 
algunos elementos que podrían estar configurando la autoestima de las mujeres y que por tanto pueden ser claves para diseñar herramientas para el fomento de la autoestima. Estos elementos son:

1. La conciencia de la opresión. La ausencia de ésta hace interpretar los daños de la autoestima y los malestares de forma intrapersonal, como una anomalía del Yo, y sintiendo, por tanto, inferioridad y culpa. En este sentido, la conciencia de género se presenta como herramienta fundamental para la autoestima. Permite comprender que ese sentimiento de anomalía no responde a un defecto real del Yo, sino que es producto de una socialización que hace sentir de ese modo a las mujeres en su conjunto. Ese nuevo marco de interpretación influye en el aspecto valorativo del Yo, fortaleciendo la autoestima. Esta conciencia no solo debe conllevar la conciencia de género sino de todas las opresiones sufridas y su consecuencias sociales y subjetivas. Solo de este modo se evitará homogeneizar las experiencias de las mujeres invisibilizando fuentes de opresión como la clase, la raza o la diversidad funcional, y podremos crear herramientas del fomento de la autoestima realmente inclusivas.

2. El Yo ideal. Este aspecto de la autoestima tiene dos dimensiones: las expectativas sociales y los deseos propios de ser. En el seno del patriarcado, los estereotipos y expectativas con que se carga socialmente a las mujeres tienen dos características fundamentales: son inalcanzables, como los cánones de belleza y cumplir con las contradicciones de la sobremodernidad, y son indeseables en el sentido de que son menos valorados socialmente que aquellos deseables en los hombres. Cabe señalar que, además, no son iguales para todas las mujeres, ya que dependen de las intersecciones de las desigualdades. Por su parte, los deseos propios de ser se ven reprimidos por considerarse, en muchas ocasiones, impropios de las mujeres, o de determinadas mujeres.

El Yo ideal se fortalece desde el feminismo a través de las referentas, ya que ellas descubren que otras formas de ser mujer, al margen de los estereotipos patriarcales, son posibles y se dan permiso para serlo. Permiten diseñar un propio Yo ideal, que se define no por cumplir las expectativas sociales sino por la propia autoestima fortalecida. 
3. La complejidad del Yo. Este elemento interfiere en la autoestima de aquellas mujeres, especialmente blancas de clase media y que sobrepasan la mediana edad, que han sido relegadas al seno del hogar. No es un factor generalizable ya que muchas mujeres cuentan con diferentes ámbitos vitales en lo que desempeñarse personalmente, aunque no todos tengan la misma carga para la autoestima, dependiendo esto de la medida en que estén valorados socialmente.

La complejidad del Yo mejora cuando existen varios ámbitos de desarrollo personal, pero siempre y cuando éstos sean valorados como tales. A ello contribuye el refuerzo y reconocimiento por parte de las/os otras/os, así como la conciencia feminista en tanto en cuanto permite comprender el Yo en

En definitiva, es necesario seguir estudiando la configuración de la autoestima en las mujeres y cuáles son los mecanismos de fortalecimiento que pueden ponerse en marcha. Esto se hace especialmente necesario en el caso de las mujeres con discriminaciones interseccionadas ya que la bibliografía al respecto es particularmente escasa. Aun así, podemos contar con algunos elementos a partir de los cuales seguir comprendiendo y fortaleciendo la autoestima de las mujeres como pilar básico para el necesario empoderamiento que consiga transformar el sistema actual en otro más justo, donde se legitimen subjetividades fortalecidas, igualitarias, libres.

\section{Referencias bibliográficas}

Bardwick, Judith M. 1986. Psicología de la mujer. Madrid: Alianza.

Berger, John. 2016. Modos de ver. Barcelona: Gustavo Gili.

Bourdieu, Pierre. 2016. La dominación masculina. Barcelona: Anagrama.

Cano, Tatiana Andrea y Arroyave, Orlando. 2014. "Procesos de empoderamiento de mujeres: subjetivación y transformaciones en las relaciones de poder". Revista Virtual Universidad Católica del Norte, 42: 94-110 http://www.redalyc.org/pdf/1942/194230899007.pdf [15 de septiembre de 2017].

Cruz Pérez, María del Pilar. 2012. "Teoría feminista y discapacidad: un complicado encuentro en torno al cuerpo". Géneros, 12: 51-71. 
http://revistasacademicas.ucol.mx/index.php/generos/article/view/634 [17 de septiembre de 2017].

Davis, Angela. 2004. Mujeres, raza y clase. Madrid: Akal.

De Beavoir, Simone. 1988. La Mujer Rota. Barcelona: Círculo de Lectores.

De Miguel, Ana. 2016. Neoliberalismo Sexual. Madrid: Cátedra.

Dio Bleichmar, Emilce. 1993. "La depresión en la mujer". Cuerpo y subjetividad femenina. Ed. María Asunción González de Chávez. Madrid: Siglo XXI. 263278.

Fascioli, Ana. 2010. "Ética del cuidado y ética de la justicia en la teoría moral de Carol Gilligan”. ACTIO, 12: 41-57.

Friedan, Betty. 2017. La mística de la feminidad. Madrid: Cátedra.

González de Chávez, María Asunción. 1993. Cuerpo y subjetividad femenina. Madrid: Siglo XXI.

González, Carmen y Lucía Valdueza. 1993. "Grupos terapéuticos de mujeres". Cuerpo y subjetividad femenina. Ed. María Asunción González de Chávez. Madrid: Siglo XXI. 279-288.

hooks, bell. 2004. "Mujeres negras. Dar forma a la teoría feminista". Otras inapropiables. VV.AA. Madrid: Traficantes de Sueños. 33-50.

Jabardo, Mercedes. 2012. Feminismos Negros: Una Antología. Madrid: Traficantes de Sueños.

Lagarde, Marcela. 2000. Claves feministas para la autoestima de las mujeres. Madrid: Horas y Horas.

Lerner, Gerda. 1986. The Creation of Patriarchy. New York: Oxford.

Levins, Aurora. 2004. "Intelectual orgánica certificada". Otras inapropiables. VV.AA. Madrid: Traficantes de Sueños. 63-70.

Linville, Patricia. 1985. "Self-Complexity and Affective Extremity: Don't Put All of Your Eggs". One Cognitive Basket. Social Cognition, 3: 94-120. http://guilfordjournals.com/doi/10.1521/soco.1985.3.1.94 [17 de septiembre de 2017].

MacMullin, Jullie Ann y John Cairney. 2004. "Self-esteem and the intersection of age, class and gender". Journal of aging studies, 18: 75-90.

Millet, Kate. 1995. Política Sexual. Madrid: Ediciones Cátedra.

Mizrahi, Liliana. 1990. Las mujeres y la culpa. Buenos Aires: Grupo Editor Latinoamericano. 
Ortega, Pedro; Mínguez, Ramón y Rodes, María Luisa. 2000. “Autoestima: un nuevo concepto y su medida". Teoría de la Educación. 12: 45-66. https://dialnet.unirioja.es/servlet/articulo?codigo $=151717$ [25 de septiembre de 2017].

Salem, Sara. 2014. "Feminismo islámico, interseccionalidad y decolonialidad". Tabula Rasa, 21: 111-122 https://www.revistatabularasa.org/numero21/feminismoislamico-interseccionalidad-y-decolonialidad/ [24 de septiembre de 2017].

Senent Ramos, Marta 2014. "Erotismo y seducción en mujeres con diversidad funciona". Dossiers Feministes, 18: 181-195. http://www.erevistes.uji.es/index.php/dossiers/article/view/1234/1251 [25 de septiembre de 2017].

Steinem, Gloria. 1995. La revolución desde dentro. Barcelona: Anagrama.

Tiggeman, Marika y Stevens, Claire. 1999. "Weight concern across the life-span: Relationship to self-esteem and feminist identity". International Journal of Eating Disorders, 26: 103-106.

Varela, Nuria. 2017. Cansadas. Barcelona: Ediciones B. 